\title{
The Influence of Solution Treatment on Structure and Performance of AZ91 Magnesium Alloy
}

\author{
Zhijun $\mathrm{Pu}^{1, \mathrm{a}}$, Hening $\mathrm{Liu}^{2}$, Kui Zhang ${ }^{2}$, Xinggang $\mathrm{Li}^{2}$ \\ ${ }^{1}$ institute of Mechanical Manufacturing Technology, China Academy of Engineering Physics, \\ Mianyang 621999, China \\ ${ }^{2}$ State Key Laboratory for Fabrication and Processing of Non-ferrous Metals, General Research \\ Institute for Non-ferrous Metals, Beijing 100088, China \\ aemail:329645634@qq.com
}

Keywords: AZ91; Solid Solution; Ageing; Corrosion; Mechanical Properties

\begin{abstract}
By means of XRD analysis, metallographic structure observation, mechanics performance testing and corrosion experiment, this paper studied the influence of solution treatment on structure and performance of AZ91 magnesium alloy. Results indicated that a large quantity of eutectic structures existed in AZ91 alloy under cast condition, and its main second phase was $\beta-\mathrm{Mg}_{17} \mathrm{Al}_{12}$; after solution treatment, eutectic structures decomposed, there was certain residue of dissociated eutectic structures, and comprehensive mechanical properties and corrosion resisting property of alloy were somewhat improved.
\end{abstract}

\section{Introduction}

As the lightest structural material, magnesium alloy has been increasingly applied to fields like aerospace. According to different shaping forms, magnesium alloy can be divided into casting magnesium alloy and wrought magnesium alloy[1][2][3]. For ordinary casting magnesium alloy, as its process is relatively simple with relatively low cost and extensive application, its development is superior to wrought magnesium alloy[4][5]. As casting defects like mingling cavities will easily appear inside casting magnesium alloy, its strength and corrosion resisting property are relatively poor. Conventional methods of improving corrosion resistance of casting magnesium alloy are alloying and heat treatment, relevant users are quite sensitive to the cost elevation brought by the former, hence, on the condition that no auxiliary element is added, using characteristics of alloying elements of mature mark and adjusting heat treatment system so as to improve corrosion resistance of alloy is an important research orientation at present[6][7]. As one of the most mature casting magnesium alloy, relevant studies and applications of AZ91 have already been very extensive. And alloys deriving from it also have certain application prospect[8]. This paper planned to study the influence of heat treatment system on AZ91 alloy performance, which could provide more theoretical basis and experimental data for expanding application of this alloy[9].

\section{Experiment}

Magnesium alloy selected in this experiment was commercial casting AZ91D magnesium alloy, it became target alloy through gravity casting after re-fusion, and its components were as shown in Table1.

Table.1. The composition of AZ91 alloys

\begin{tabular}{cccccc}
\hline Alloy & $\mathrm{Al}$ & $\mathrm{Zn}$ & $\mathrm{Mn}$ & Impurity & $\mathrm{Mg}$ \\
\hline AZ91 & 9.0 & 0.6 & 0.15 & $\leq 0.04$ & Balance \\
\hline
\end{tabular}




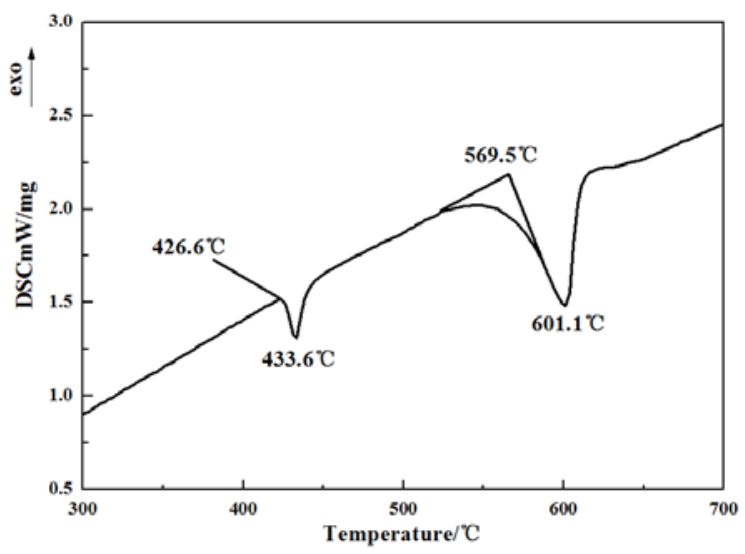

Fig.1. DSC curve of casting AZ91 magnesium alloy

Figure1 was temperature-rising DSC curve of AZ91 magnesium alloy, starting melting temperature of low-melting point phase was $426.6^{\circ} \mathrm{C}$, according to empirical formula $\mathrm{T}_{\text {solid }}$ solution $=0.9-0.95 \mathrm{~T}_{\mathrm{m}}$, solution temperature of alloy should not be higher than $400^{\circ} \mathrm{C}$, so final solution temperatures were set as $360^{\circ} \mathrm{C}, 375^{\circ} \mathrm{C}$ and $390^{\circ} \mathrm{C}$, and holding time should not exceed $24 \mathrm{~h}$ at most. Hardness test of alloy was implemented on HBS-62.5 small-load Brinell hardness tester, and loading time was 30s; hardness test was repeated for five times, and average value was taken. Normal temperature mechanical properties of the material were tested on Shimadzu testing machine and the speed was $2 \mathrm{~mm} / \mathrm{min}$. Polarization curves of alloy were measured on RST5200 electrochemical workstation, reference electrode was calomel electrode, medium was $3.5 \% \mathrm{NaCl}$ solution and temperature was tested as indoor temperature. Microstructure observation was made under CarlZeiss Axiovet 200MAT metallograpic microscope and JSM microscope.

\section{Results and Analysis}

\section{Alloy under cast condition}

Figure2 was AZ91 magnesium alloy microstructure under cast condition and XRD atlas. It could be seen that the alloy mainly consisted of $\alpha$-Mg matrix and skeleton-shaped eutectic structure (Figure2-a), during solidification process, solute atoms gathered at solidification front, when composition of eutectic point was reached, it underwent alternate solidification with matrix and thus forming eutectic structure. It could be seen from XRD atlas (Figure2-b) that the main second phase in alloy was $\beta-\mathrm{Mg}_{17} \mathrm{Al}_{12}$, initial solution temperature of this phase was $427^{\circ} \mathrm{C}$ which was approximate to that in DSC curve. Thus it could be seen that low-melting point endothermic peak in DSC curve was mainly generated by $\beta-\mathrm{Mg}_{17} \mathrm{Al}_{12}$ phase decomposition.

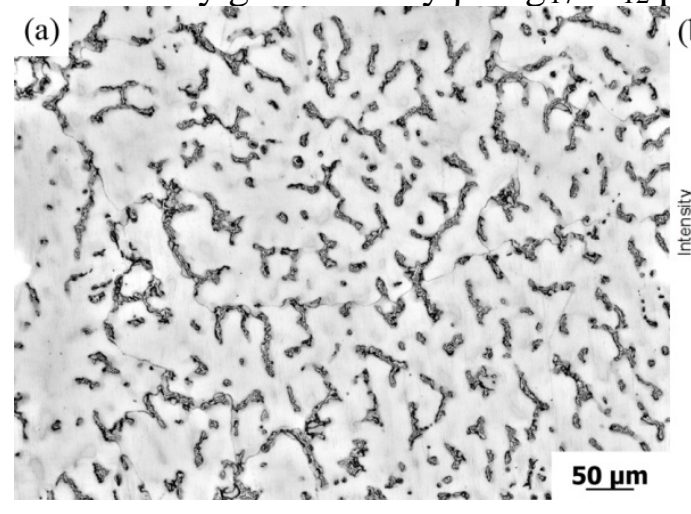

(a) Microstructure of as cast AZ91 alloy (b)

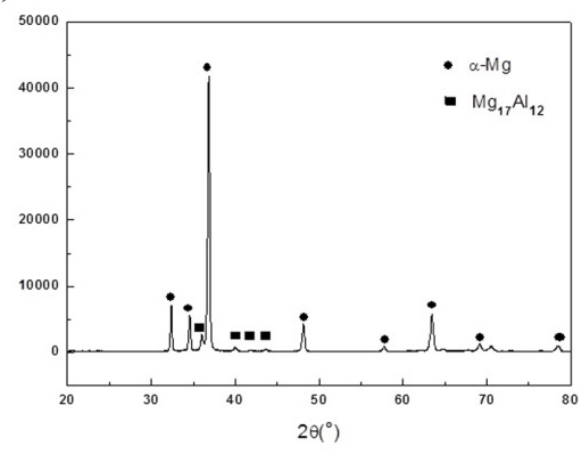

(b) XRD analysis of as cast AZ91 alloy

Fig.2.Microstructure and XRD analysis of as cast AZ91 alloy

\section{Alloy solution treatment}

Hardness of alloy after solid solution was as shown in Figure3. It could be seen that under the same temperature, as time went by, hardness of alloy would firstly decrease and then tended to be 
balanced without obvious rising, namely from the angle of hardness, there was no significant strengthening effect of solid solution; within the same period, hardness of alloy would decrease as temperature increased. There was certain casting stress in solidification process of alloy, when alloy began solution treatment, casting stress would then be released and hardness obviously decreased. In the meantime, under certain temperature, eutectic structure of alloy decomposed, solute atoms returned to matrix and formed subrogating solid solution, thus causing lattice distortion, improving difficulty of dislocation motion and increasing intensity of alloy; grain size of alloy would obviously enlarge as solution temperature increased, it could be known from Hall-Petch relationship that intensity of magnesium alloy depended on grain size to a great extent, and oversize grain would surely result in decreasing alloy intensity. Hardness of alloy would finally be decided jointly by solution strengthening, release of casting stress and weakening caused by grain growth. It could be seen from the figure that weakening effect was obviously stronger than strengthening effect, and final hardness of alloy presented decreasing tendency.

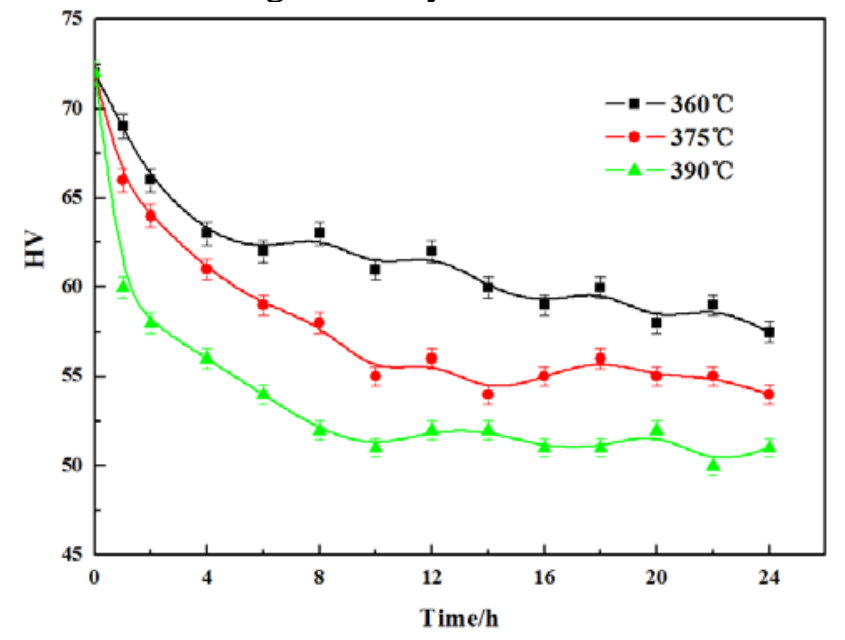

Fig.3.Curves of hardness with time of AZ91 alloy

Influence of solution treatment on AZ91 alloy structure was as shown in Figure4. It could be seen that under $360^{\circ} \mathrm{C}$ and $375^{\circ} \mathrm{C}$, after $24 \mathrm{~h}$ treatment, a large quantity of undissolved second phases still existed, which indicated that above two temperature values were low; under $390^{\circ} \mathrm{C}$, after 12 , many second phases decomposed, but a certain amount of undissolved phases still existed. After 24h treatment, decomposed quantity of second phases of alloy didn’t increase obviously. In Mg-Al alloy, there were usually two eutectic types: dissociated eutectic and stratiform eutectic, it could be seen from metallographic phase that undecomposed second phases were mainly second phases in dissociated eutectic, the main reason why it was not easy for this kind of second phases to experience decomposition might be that solute atoms presented aggregating shape, and compared with stratiform eutectic, there was no path for generating mutual diffusion. Hence, it was difficult to decompose. In terms of hardness and metallograpic phase, in this test, alloy reached extreme solubility under this condition after $12 \mathrm{~h} 390^{\circ} \mathrm{C}$ heat preservation. 


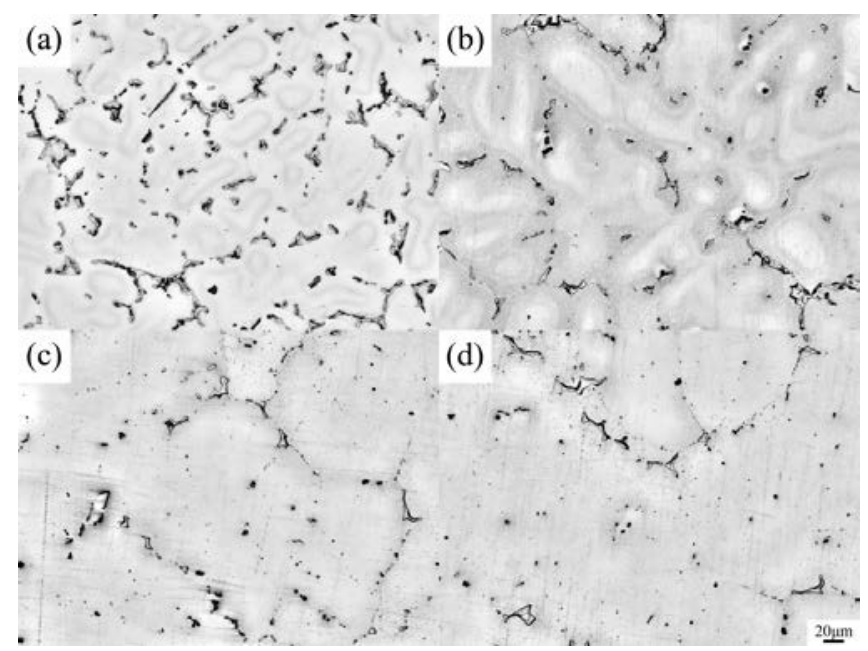

Fig.4.Effect of solid solution treatment on Microstructure of AZ91 alloy

\section{Mechanical properties of alloy}

Mechanical properties of alloy before and after solid solution were as shown in Figure5. It could be seen that mechanical properties of alloy under cast condition were poor, average breaking strength was only $145 \mathrm{MPa}$, and average ductility was only $3.5 \%$; after solution treatment, average breaking strength of alloy elevated to 180MPa while ductility also elevated to $6.0 \%$. Elevation of alloy intensity might resulted from two factors: effect of solution strengthened, and on the other hand, reduced solidification segregation in alloy decreased focal points of stress and improved coordinating deformability between grains, thus finally improving comprehensive mechanical properties of alloy; in the meantime, as after solution treatment, grain size of alloy obviously grew, alloy would be weakened, it could be known by combining alloy hardness result that the effect of solution strengthening effect on improving alloy intensity was lower than effect of elimination of solidification segregation on improvement of coordinating deformability between grains, namely decomposition of eutectic structure reduced crack sources of alloy and improved mechanical properties of alloy.

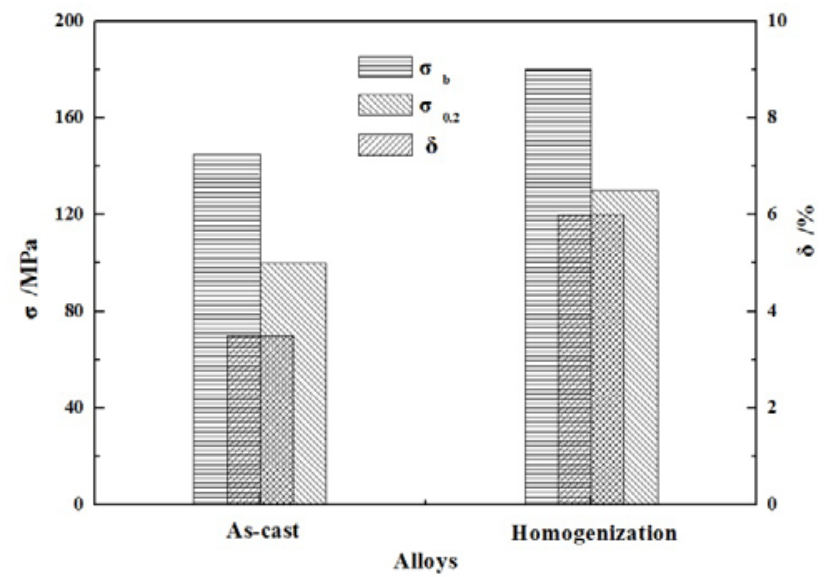

\section{Corrosion resisting property of alloy}

Fig.5.Mechanical properties of alloys

The influence of solution treatment on electrode potential of AZ91 alloy was as shown in Figure6. It could be seen that when the temperature was certain, as solution time passed by, electrode potential of alloy moved toward direction of positive potential, which indicated improved corrosion resisting property of alloy; when the time was certain, electrode potential of alloy increased as solution temperature increased. Increasing electrode potentials indicated improvement of corrosion resisting property of alloy. 


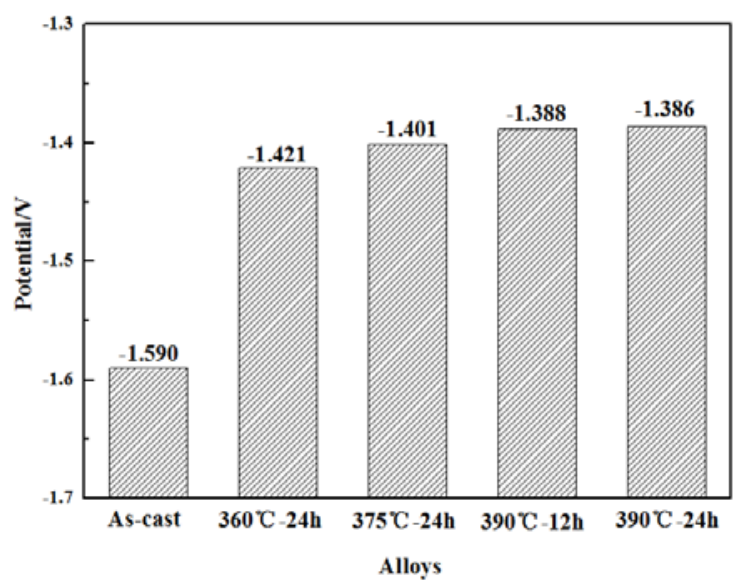

Fig.6. Effect of solid solution treatment on electrode potential of AZ91 alloy

Oxidation film formed by magnesium alloy in air was not complete, and it didn't resist corrosion. In the meantime, as there were many eutectic structures in magnesium alloy under cast condition, there were many places in which primary battery reaction occurred, and then corrosion rate of alloy was aggravated. As volume of $\mathrm{Cl}^{-}$ion in socking medium was small, it would easily penetrate surface oxidation film of alloy, thus damaging oxidation film which was originally incomplete and accelerating corrosion. After solution treatment, eutectic structures in alloy reduced, places where primary battery reaction occurred reduced, and corrosion resistance of ally was improved. In the meantime, as aluminum atoms entered alloy through solution, oxidation film formed by aluminum was compact, which could effectively improve corrosion resistance of alloy. It could be known from experimental results that solution degree of alloy decided its corrosion resistance, the higher the solution degree was, the stronger the corrosion resistance of alloy would be.

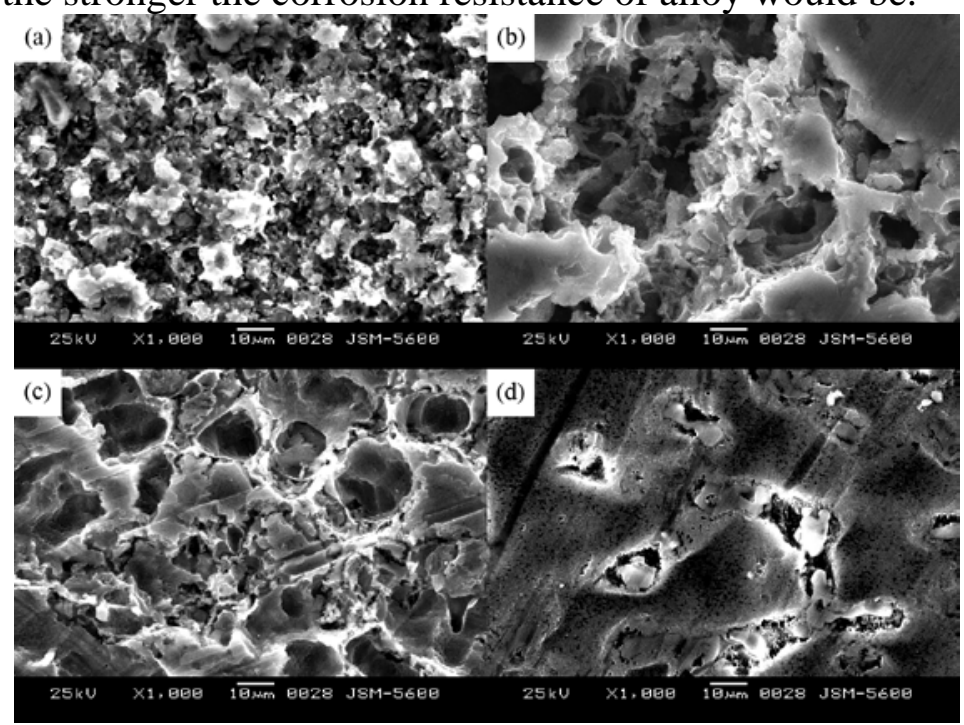

(a)as cast, (b) $390^{\circ} \mathrm{C} \times 8 \mathrm{~h}$, (c) $390^{\circ} \mathrm{C} \times 16 \mathrm{~h},(\mathrm{~d}) 390^{\circ} \mathrm{C} \times 24 \mathrm{~h}$

Micro corrosion morphology of alloy after solid solution treatment

Figure7 was about microscopic corrosion morphology of alloy after solution treatment, it could be known that as solution time lengthened, area of alloy in which corrosion occurred gradually reduced, the place where eutectic structure contracted matrix limitedly experienced corrosion, thus it was indicated that after solution time lengthened, eutectic structures were eliminated, quantity of primary batteries decreased, which could effectively improve corrosion resistance of alloy, there was obvious potential difference between matrix and eutectic structures, so it would be corroded with a priority. 


\section{Conclusion}

(1)AZ91 alloy under cast condition consisted of $\alpha$-Mg matrix and skeleton-shaped eutectic structures, and its main second phase was $\beta-\mathrm{Mg}_{17} \mathrm{Al}_{12}$.

(2) After solution treatment, eutectic structures of alloy decomposed, there was certain residue of dissociated eutectic structures; hardness of alloy was influenced by solution temperature and time. When the temperature was certain, hardness would first decrease with time and then tended to be balanced; when time was certain, hardness of alloy would decrease as solution temperature increased.

(3) After solution treatment, comprehensive mechanical properties of alloy were improved, breaking strength reached $180 \mathrm{MPa}$, while ductility reached $6 \%$, in the meantime, corrosion resisting property of alloy also correspondingly increased, eutectic structures decomposed, and the main reason for improvement of corrosion resistance was reduced places where primary battery reaction occurred.

\section{References}

[1] Huisheng CAI, et al. "Effects of cerium on as-cast microstructure of AZ91 magnesium alloy under different solidification rates." Journal of rare earths (English Edition) 34.7(2016):736-741.

[2] Wang, Cui Ju, et al. "Competition behavior of the strengthening effects in as-extruded AZ91 matrix: Influence of pre-existed $\mathrm{Mg} 17 \mathrm{Al}$ 12, phase."Materials Science \& Engineering A 656(2016):102-110.

[3] Liu, Cancan, et al. "Characterization and corrosion behavior of plasma electrolytic oxidation coated AZ91-T6 magnesium alloy." Surface \& Coatings Technology (2016).

[4] Strzelecka, M., et al. "Surface modification of the AZ91 magnesium alloy." Archives of Civil \& Mechanical Engineering 15.4(2015):854-861.

[5] Al-Zubaydi, Ahmed S. J., et al. "Superplastic behaviour of AZ91 magnesium alloy processed by high-pressure torsion." Materials Science \& Engineering A 637(2015):1-11.

[6] Johnston, Sean, Z. Shi, and A. Atrens. "The influence of $\mathrm{pH}$ on the corrosion rate of high-purity Mg, AZ91 and ZE41 in bicarbonate buffered Hanks' solution." Corrosion Science 101 (2015) : 182-192.

[7] Wang, Lei, T. Shinohara, and B. P. Zhang. "XPS study of the surface chemistry on AZ31 and AZ91 magnesium alloys in dilute $\mathrm{NaCl}$ solution."Applied Surface Science 256. 256 (2010) : 5807-5812.

[8] Srinivasan, A., et al. "Creep Behavior of AZ91 Magnesium Alloy $\hat{\sim}^{2}$."Procedia Engineering 55.6 (2013):109-113.

[9] Esgandari, B. Amir, et al. "The effect of $\mathrm{Ca}$ and $\mathrm{RE}$ elements on the precipitation kinetics of $\mathrm{Mg}$ $17 \mathrm{Al}$ 12, phase during artificial aging of magnesium alloy AZ91." Materials Science \& Engineering A528. 15 (2011):5018-5024. 